\title{
Coexistence of, and Competition between, Superconductivity and Charge-Stripe Order in $\mathrm{La}_{1.6-x} \mathrm{Nd}_{0.4} \mathrm{Sr}_{x} \mathrm{CuO}_{4}$
}

\author{
J. M. Tranquada, ${ }^{1}$ J. D. Axe,${ }^{1}$ N. Ichikawa, ${ }^{2}$ A. R. Moodenbaugh, ${ }^{1}$ Y. Nakamura, ${ }^{2}$ and S. Uchida ${ }^{2}$ \\ ${ }^{1}$ Brookhaven National Laboratory, Upton, New York 11973 \\ ${ }^{2}$ Department of Superconductivity, The University of Tokyo, Bunkyo-ku, Tokyo 113, Japan
}

(Received 6 August 1996)

\begin{abstract}
Previously we have presented evidence for stripe order of holes and spins in $\mathrm{La}_{1.6-x} \mathrm{Nd}_{0.4} \mathrm{Sr}_{x} \mathrm{CuO}_{4}$ with $x=0.12$. Here we show, via neutron diffraction measurements of magnetic scattering, that similar order occurs in crystals with $x=0.15$ and 0.20 . Zero-field-cooled magnetization measurements show that all three compositions are also superconducting, with the superconducting transition temperature increasing as the low-temperature staggered magnetization decreases. These results directly demonstrate an intimate connection between stripe correlations and superconductivity. [S0031-9007(96)02195-3]
\end{abstract}

PACS numbers: 74.72.Dn, 71.45.Lr, 75.50.Ee, 75.70.Kw

Neutron scattering studies [1-7] of dynamical magnetic correlations in superconducting $\mathrm{La}_{2-x} \mathrm{Sr}_{x} \mathrm{CuO}_{4}$ have provided important clues to the nature of electronic correlations within the doped $\mathrm{CuO}_{2}$ planes. The low-energy magnetic scattering, which is characterized by the twodimensional antiferromagnetic wave vector $\mathbf{Q}_{\mathrm{AF}}=\left(\frac{1}{2}, \frac{1}{2}\right)$ (measured in units $2 \pi / a$ ) at low doping, shifts to positions $\left(\frac{1}{2} \pm \epsilon, \frac{1}{2}\right)$ and $\left(\frac{1}{2}, \frac{1}{2} \pm \epsilon\right)$, with $\epsilon \approx x$ for $x>0.05$ [7]. In one common interpretation [8-11], the incommensurate peaks are viewed as the dynamical response of a spatially uniform electron liquid with a nearly nested Fermi surface. From a rather different perspective, the $Q$-dependent structure is taken as evidence for spatial inhomogeneity associated with charge segregation [12-14] or charge-density-wave correlations [15-18]. Evidence for the latter picture is provided by our recent discovery $[19,20]$ of incommensurate charge and spin order in $\mathrm{La}_{1.6-x} \mathrm{Nd}_{0.4} \mathrm{Sr}_{x} \mathrm{CuO}_{4}$ with $x=0.12$; however, given the claim [21] that bulk superconductivity is absent at this composition, one might choose to argue that these results are not directly relevant to the case of superconducting samples.

To test the relationship between charge-stripe order and superconductivity, we have now investigated two other $\mathrm{Sr}$ concentrations, $x=0.15$ and 0.20 . Our neutron diffraction measurements on single-crystal samples reveal elastic incommensurate magnetic peaks for both compositions, thus demonstrating the presence of charge-stripe order. Since the $x=0.20$ crystal was known to be superconducting from previous work [22], we decided to check the $x=0.12$ and 0.15 crystals for superconductivity as well. To our surprise, zero-field-cooled susceptibility measurements exhibit a bulk shielding signal for all three compositions. Since both the incommensurate peak splitting $\epsilon$ and the superconducting transition temperature vary with $x$, the results strongly suggest a local coexistence of superconductivity and stripe order. The fact that $T_{c}$ decreases as the staggered magnetization increases indicates that these two types of order compete with one another [23]. Fur- thermore, since the variation of $\epsilon$ with $x$ in the Nd-doped crystals is essentially identical to that obtained from recent inelastic measurements [7] on crystals of $\mathrm{La}_{2-x} \mathrm{Sr}_{x} \mathrm{CuO}_{4}$, it seems inescapable that dynamical charge-stripe correlations are present in the optimally doped material.

The crystals studied in this work were grown at the University of Tokyo using the traveling-solvent floatingzone method. The transport properties of the $x=0.12$ and 0.20 compositions were reported several years ago [22]; the $x=0.15$ and further $x=0.12$ crystals were grown more recently. The neutron diffraction measurements on the $x=0.15$ and 0.20 crystals were performed on triple-axis spectrometers at the High Flux Beam Reactor, Brookhaven National Laboratory, utilizing cryostats and spectrometer conditions similar to those used in the previous work on $x=0.12$, which is described in detail elsewhere $[19,20]$.

Scans through the magnetic peaks at $\mathbf{Q}=\left(\frac{1}{2} \pm \epsilon, \frac{1}{2}, 0\right)$ are shown in Fig. 1. Sharp elastic peaks (with resolutionlimited widths in these coarse-resolution scans) are found for all three $\mathrm{Sr}$ concentrations. The peak splitting parameter $\epsilon$ is distinctly different in each sample, and clearly increases with $x$. The temperature dependences of the magnetic peak intensities (normalized to sample volume) are presented in Fig. 2. Both the ordering temperature and the relative intensity (proportional to the square of the staggered magnetization) decrease with $x$. The sharp upturn in intensity at low $T$ that is apparent for the $x=0.20$ sample is identical to that found previously for $x=0.12$ $[19,20]$ and is due to ordering of the Nd moments via coupling to the $\mathrm{Cu}$ ions. The $\mathrm{Nd}$ ordering provides a useful amplification of the $\mathrm{Cu}$ order.

Unfortunately there is no such incidental amplification of the charge-order peaks, which were already quite weak for $x=0.12$. An extremely weak signal was detected at the expected position $(2+2 \epsilon, 0,0)$ for the $x=0.15$ crystal at $10 \mathrm{~K}$, but it was not practical to determine its temperature dependence. No search for a charge-order peak in the $x=0.20$ sample was even attempted due to 


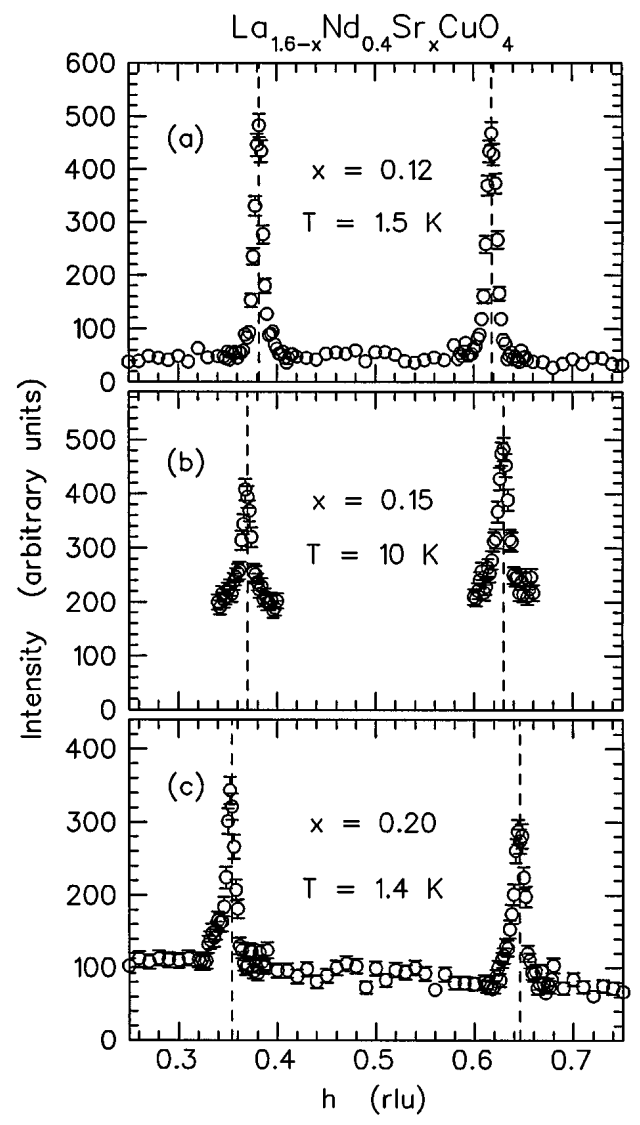

FIG. 1. Scans along $\mathbf{Q}=\left(h, \frac{1}{2}, 0\right)$ through the magnetic peaks at $h=\frac{1}{2} \pm \epsilon$ measured on crystals of $\mathrm{La}_{1.6-x} \mathrm{Nd}_{0.4} \mathrm{Sr}_{x} \mathrm{CuO}_{4}$ with (a) $x=0.12$, (b) $x=0.15$, and (c) $x=0.20$. Note that the measurements are not all at the same temperature.

the small size of the crystal $\left(\sim 0.05 \mathrm{~cm}^{3}\right)$ and to the weakness of the magnetic signal. Nevertheless, even without a direct observation of charge order (or, rather, the corresponding lattice modulation to which neutrons are sensitive), a modulation of the charge density is implied by the incommensurate magnetic order. The argument behind this assertion is as follows. The magnetic incommensurability indicates that there exists a modulation of either the spin orientations (spiral order) or the spin density [24]; a combination of these two is also possible. We have argued elsewhere [20] that the secondary ordering of the $\mathrm{Nd}$ moments is incompatible with perfect spiral order of the $\mathrm{Cu}$ spins within a plane; therefore, there must be a spindensity-wave component to the order. Symmetry allows a spin modulation with wave vector $\mathbf{q}$ to couple to a charge modulation at $\mathbf{2 q}$. It follows that a charge-density modulation must be present; the only real issues concern the magnitude of the modulation and the driving mechanism. In the case of $x=0.12$, the neutron diffraction data indicate that the order is driven by the charge $[19,20,24]$. There is nothing to suggest that the physics is any different in the $x=0.15$ and 0.20 crystals.

To test for superconductivity in the crystals (or pieces thereof), the bulk magnetic susceptibility was measured

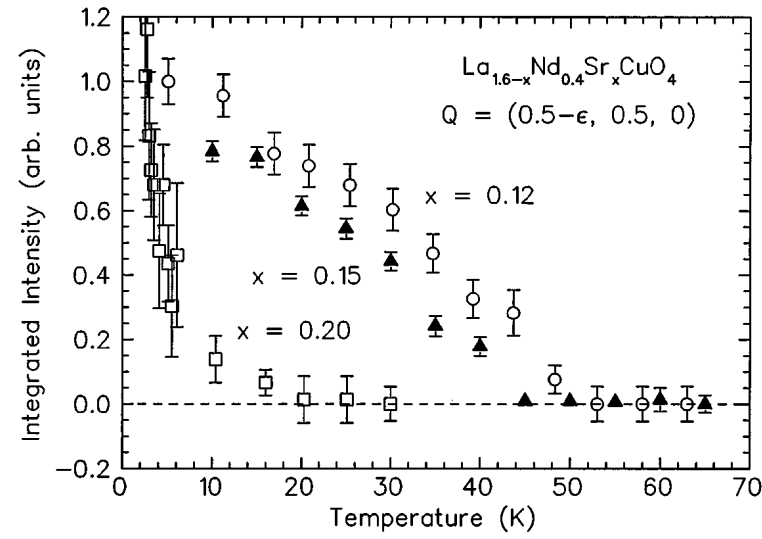

FIG. 2. Temperature dependence of the incommensurate magnetic peak intensity for crystals with $x=0.12$ (circles; Ref. [20]), 0.15 (filled triangles), and 0.20 (squares). Intensities are normalized for sample volume.

with a SQUID (superconducting quantum-interference device) magnetometer, using a magnetic field in the range of 1-5 G. Attempts to measure the Meissner effect (by cooling in a magnetic field) yielded a weak paramagnetic upturn at $T_{c}$. On the other hand, measurements performed after cooling in zero field (see Fig. 3) give a shielding signal $>100 \%$ (without correction for demagnetization). We believe that the shielding results provide reliable evidence of bulk superconductivity in the crystals. The variation of $T_{c}$ with $x$ argues against associating the superconductivity with an impurity phase.

There have been disagreements in the literature $[21,25]$ concerning the existence of bulk superconductivity in $\mathrm{La}_{1.6-x} \mathrm{Nd}_{0.4} \mathrm{Sr}_{x} \mathrm{CuO}_{4}$ for $x \lessgtr 0.2$. In particular, Büchner et al. [21] have argued against bulk superconductivity on the basis of Meissner-effect and specific-heat measurements. Concerning the specific heat, it is observed in the cuprates that the jump at $T_{c}$ rapidly becomes

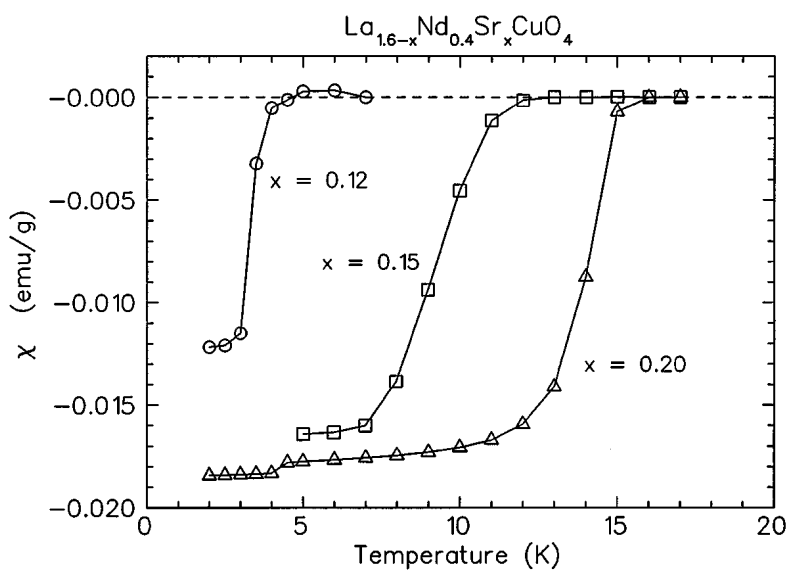

FIG. 3. Bulk magnetic susceptibility measured after cooling in zero field, for crystals with $x=0.12,0.15$, and 0.20 . The kink at $4 \mathrm{~K}$ for $x=0.20$ is attributed to hysteresis in the magnet. 
smeared as doping conditions deviate from optimal [26]; hence, it is not surprising if a superconducting transition is not readily apparent in specific-heat measurements on a sample with a severely depressed $T_{c}$. As a check on the present single-crystal results, a series of ceramic samples was prepared. Superconducting ceramic samples ground to a fine powder yield volume fractions (in both Meissner and shielding measurements) comparable to those found in $\mathrm{La}_{2-x} \mathrm{Sr}_{x} \mathrm{CuO}_{4}$ [27]. The variation of $T_{c}$ with $x$ is compared with the single-crystal results in Fig. 4(c). The behaviors are reasonably consistent. The values of $T_{c}$ for single crystals of $\mathrm{La}_{2-x} \mathrm{Sr}_{x} \mathrm{CuO}_{4}$ studied recently by $\mathrm{Ya}-$ mada et al. [7] are also included (open circles).

The reduction of $T_{c}$ induced by the $\mathrm{Nd}$ substitution is correlated with a modification of the low temperature tilt pattern of the $\mathrm{CuO}_{6}$ octahedra [25]. The $\mathrm{Nd}$ causes a change in the tilt direction from [110], as in the low-temperature-orthorhombic (LTO) phase, to [100], characteristic of the low-temperature-tetragonal

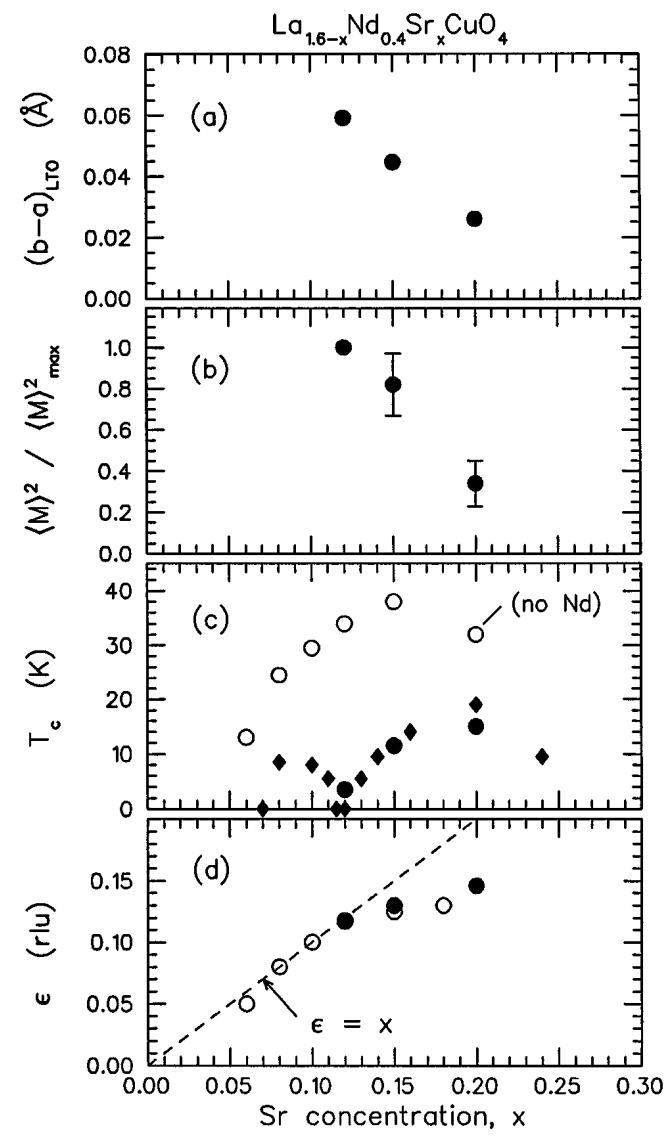

FIG. 4. Comparison of results as a function of $\mathrm{Sr}$ concentration: (a) difference between $a$ and $b$ lattice parameters in the LTO phase measured just above the transition to the LTT phase, (b) square of the low-temperature staggered magnetization, normalized to the $x=0.12$ result, (c) superconducting transition temperature, and (d) incommensurate splitting $\epsilon$. Filled symbols: $\mathrm{La}_{1.6-x} \mathrm{Nd}_{0.4} \mathrm{Sr}_{x} \mathrm{CuO}_{4}$; open symbols: $\mathrm{La}_{2-x} \mathrm{Sr}_{x} \mathrm{CuO}_{4}$ (Ref. [7]). Circles: single-crystal samples; diamonds: ceramic samples.
(LTT) phase, with the transition occurring at a temperature of roughly $70 \mathrm{~K}$. A coupling between the tilt modulation and the charge-stripe correlations is possible only when the tilts have a [100] orientation, parallel to the charge modulation. One might expect that the degree to which the charge modulations can be pinned would depend on the amplitude of octahedral tilts. Büchner et al. [21] have shown that a useful measure of the tilt amplitude (or actually its square) is the maximum difference between $a$ and $b$ lattice parameters in the LTO phase. The values of $b-a$ measured by neutron diffraction on our Nd-doped crystals are shown as a function of Sr concentration in Fig. 4(a); for comparison, the square of the staggered magnetization (low-temperature magnetic peak intensity normalized relative to the $x=0.12$ result) is presented in (b). The strength of the magnetic order is clearly correlated with the size of the tilt modulation, consistent with the pinning argument. The magnitude of $T_{c}$ reduction is also correlated with the tilt modulation. Superconductivity and stripe order compete with each other, but also coexist.

Of course, the hole concentration also varies with $x$, and this is reflected in the variation of the magneticpeak-splitting parameter $\epsilon$, as indicated by the filled circles in Fig. 4(d). The open circles are the results of inelastic measurements on crystals of $\mathrm{La}_{2-x} \mathrm{Sr}_{x} \mathrm{CuO}_{4}$ by Yamada et al. [7]. The trends with $x$ are essentially identical, implying that the nature of the instantaneous correlations in the two systems is the same. The recent observation [6] that high-energy spin fluctuations in $\mathrm{La}_{1.86} \mathrm{Sr}_{0.14} \mathrm{CuO}_{4}$ behave like damped spin waves certainly seems consistent with the presence of stripe correlations. The only significant difference between the crystals with and without $\mathrm{Nd}$ appears to be the degree of pinning of the stripe correlations. Fluctuations of the stripe correlations seem to be important for achieving a high $T_{c}$.

Theoretically, calculations by Viertiö and Rice [28] indicate that charged domain walls in a doped antiferromagnet will tend to melt due to quantum fluctuations. This result is quite consistent with experiment: No static stripe order is observed in optimally doped $\mathrm{La}_{2-x} \mathrm{Sr}_{x} \mathrm{CuO}_{4}$. Quantum melting is inhibited only when a sufficiently strong perturbation, such as that caused by $\mathrm{Nd}$ substitution, is applied. The tendency of domain walls to fluctuate has also been considered in other recent studies $[29,30]$. Fluctuating stripe correlations seem a likely explanation for the quantum critical behavior found in $\mathrm{La}_{1.86} \mathrm{Sr}_{0.14} \mathrm{CuO}_{4}$ by Aeppli et al. [5].

The spatial modulation of spin and charge densities indicated by our results could be driven either by a Fermisurface-induced charge-density-wave (CDW) instability or by frustrated phase separation. CDW order is generally stabilized by the opening of a gap about the Fermi energy. Such a gap would seem to be inconsistent with the low resistivity [22] and superconductivity in these samples; furthermore, optical measurements show no evidence for 
a gap in charge excitations down to $4 \mathrm{meV}$ [31]. On the other hand, in the frustrated-phase-separation model $[13,32]$, the chemical potential should lie within a band of mid-gap states, consistent with metallic behavior. (An alternative strong-correlation model with charge stripes is described in [33].)

To summarize, we have presented evidence that superconductivity and charge-stripe order coexist in $\mathrm{La}_{1.6-x} \mathrm{Nd}_{0.4} \mathrm{Sr}_{x} \mathrm{CuO}_{4}$, although the order parameters compete with one another. For a given $x$, the spatial modulation of the spin correlations is the same as in $\mathrm{La}_{2-x} \mathrm{Sr}_{x} \mathrm{CuO}_{4}$ [7], which indicates that the instantaneous correlations are essentially the same in the two systems. There appears to be an intimate connection between stripe correlations and superconductivity in these materials. It will be interesting to test the generality of these results in other families of cuprate superconductors.

We gratefully acknowledge stimulating discussions with R. J. Birgeneau, V. J. Emery, S. A. Kivelson, M. A. Kastner, G. Shirane, F. Wilczek, K. Yamada, and O. Zachar. We also thank K. Yamada for sharing his results prior to publication. Work at Brookhaven was carried out under Contract No. DE-AC02-76CH00016, Division of Materials Sciences, U.S. Department of Energy.

[1] S.-W. Cheong et al., Phys. Rev. Lett. 67, 1791 (1991).

[2] T. E. Mason, G. Aeppli, and H. A. Mook, Phys. Rev. Lett. 68, 1414 (1992).

[3] T. R. Thurston et al., Phys. Rev. B 46, 9128 (1992).

[4] K. Yamada et al., Phys. Rev. Lett. 75, 1626 (1995).

[5] G. Aeppli, T.E. Mason, S. M. Hayden, and H. A. Mook (to be published).

[6] S. M. Hayden et al., Phys. Rev. Lett. 76, 1344 (1996).

[7] K. Yamada et al. (to be published).

[8] N. Bulut, D. Hone, D. J. Scalapino, and N.E. Bickers, Phys. Rev. Lett. 64, 2723 (1990).

[9] Q. Si, Y. Zha, K. Levin, and J.P. Lu, Phys. Rev. B 47, 9055 (1993).
[10] P. B. Littlewood, J. Zaanen, G. Aeppli, and H. Monien, Phys. Rev. B 48, 487 (1993).

[11] T. Tanamoto, H. Kohno, and H. Fukuyama, J. Phys. Soc. Jpn. 63, 2739 (1994).

[12] U. Löw, V.J. Emery, K. Fabricius, and S. A. Kivelson, Phys. Rev. Lett. 72, 1918 (1994).

[13] S. A. Kivelson and V.J. Emery, in Proceedings of the Los Alamos Symposium on Strongly Correlated Electronic Materials, Los Alamos, New Mexico, 1993, edited by K. S. Bedell et al. (Addison-Wesley, Reading, Massachusetts, 1994), pp. 619-656.

[14] L. Chayes et al., Physica (Amsterdam) 225A, 129 (1996).

[15] J. Zaanen and O. Gunnarsson, Phys. Rev. B 40, 7391 (1989).

[16] H. J. Schulz, J. Phys. (Paris) 50, 2833 (1989).

[17] D. Poilblanc and T.M. Rice, Phys. Rev. B 39, 9749 (1989).

[18] C. Castellani, C. D. Castro, and M. Grilli, Phys. Rev. Lett. 75, 4650 (1995).

[19] J. M. Tranquada et al., Nature (London) 375, 561 (1995).

[20] J. M. Tranquada et al., Phys. Rev. B 54, 7489 (1996).

[21] B. Büchner, M. Breuer, A. Freimuth, and A.P. Kampf, Phys. Rev. Lett. 73, 1841 (1994).

[22] Y. Nakamura and S. Uchida, Phys. Rev. B 46, 5841 (1992).

[23] Y. Imry, J. Phys. C 8, 567 (1975).

[24] O. Zachar, V. J. Emery, and S. A. Kivelson (unpublished).

[25] M. K. Crawford et al., Phys. Rev. B 44, 7749 (1991).

[26] J. W. Loram, K. A. Mirza, W. Y. Liang, and J. Osborne, Physica (Amsterdam) 162C-164C, 498 (1989).

[27] T. Nagano et al., Phys. Rev. B 48, 9689 (1993).

[28] H. E. Viertiö and T. M. Rice, J. Phys. Condens. Matter 6, 7091 (1994).

[29] J. Zaanen, M. L. Horbach, and W. van Saarloos, Phys. Rev. B 53, 8671 (1996).

[30] H. Eskes, R. Grimberg, W. van Saarloos, and J. Zaanen, Phys. Rev. B 54,724R (1996).

[31] S. Tajima, Y. Nakamura, and S. Uchida (unpublished).

[32] S. A. Kivelson and V.J. Emery, Synth. Met. 80, 151 (1996).

[33] C. Nayak and F. Wilczek, Int. J. Mod. Phys. B 10, 2125 (1996). 